\title{
La expansión del parque automotor y la red vial en Mendoza: El papel de la Dirección de Puentes y Caminos en la década del '20
}

\author{
Natalia Luis ${ }^{*}$
}

Fecha de Recepción: 30 de julio 2021

Fecha de Aceptación: 25 de octubre de 2021

DOI: https://doi.org/10.46553/RGES.57.2021.p.136-160

\begin{abstract}
Resumen: El desarrollo de vías comunicacionales terrestres es esencial para la vida de la población, para facilitar la actividad comercial y para garantizar el flujo de personas e ideas. Durante las primeras décadas del siglo XX, la política vial en Mendoza tenía una estructura minúscula, estaba en manos de la Dirección de Puentes y Caminos (DPyC), dependiente del Ministerio de Obras Públicas (ley 393, 1907) y no existía un plan sistemático en relación con las vías de comunicación. La agencia estatal se fue especializando y en 1933 se creó la Dirección Provincial de Vialidad, siguiendo el modelo nacional. El objetivo de este artículo es indagar las características de la red vial en Mendoza durante la década del '20 haciendo hincapié en el papel de la DPyC. Asimismo, se analizará las vías de comunicación y los medios de transporte, ambos aspectos junto con la transformación urbana vinculada a ellos, considerando datos estadísticos, proyectos de ley relativos a la vialidad y su recepción en la prensa local a fin de observar su impacto en la opinión pública.
\end{abstract}

Palabras clave: agencia estatal; parque automotor; red vial; Mendoza.

Summary: The development of land communication routes is essential for the life of the population, to facilitate commercial activity and to guarantee the flow of people and ideas. During the first decades of the twentieth century, road policy in Mendoza had a tiny structure. It was in the hands of the Directorate of Bridges and Roads (DPyC), under the Ministry of Public Works (law 393, 1907) and there was no systematic plan in relation to the roads of communication. The state agency in charge of roads was specialized and in 1933 the Provincial Directorate of Roads was created, following the national model. The objective of this article is to investigate the characteristics of the road network in Mendoza during the decade of the '20s emphasizing the role of the DPyC. In addition, the roads and means of transport will be analysed, both aspects together with the urban transformation linked to them, considering statistical data and draft laws relating to roads and their reception in the local press in order to observe their impact on public opinion.

Keywords: state agency; vehicle fleet; road network; Mendoza.

"es indudable y esto está en la mente de todos, que una región que no tiene caminos por donde conducirse con rapidez y comodidad es una región desvalorizada en relación a otras que tienen

\footnotetext{
* Doctora en Historia. Becaria postdoctoral de INCIHUSA CONICET, Mendoza. Mail: nluis@mendozaconicet.gob.ar/ natalita812@gmail.com
} 
caminos en perfectas condiciones (...)” (Senador Gaviola, sesión legislativa del día 5 de septiembre de 1918).

\section{Introducción}

El desarrollo de vías de comunicación terrestres (calles, rutas, puentes) es esencial para la vida de la población, por un lado, para facilitar la actividad comercial y el abastecimiento, y por otro, para garantizar el flujo de personas e ideas, tanto en el interior de las provincias y el país, como en relación con los países extranjeros. Para Mendoza, provincia asentada al pie de la cordillera de Los Andes (ver ilustración 1), última antes de la frontera con Chile en el camino Buenos Aires-Santiago, y alejada de la capital nacional y del puerto atlántico, fue una preocupación central el desarrollo de caminos y la implementación de medios de transporte (entre los que en un primer momento destacó el ferrocarril) para garantizar la comunicación intra-provincial, con otras provincias argentinas y con Chile debido a su proximidad. Cabe destacar en este sentido la complementariedad que existía entre los caminos y el ferrocarril. De hecho, entre fines del siglo XIX y comienzos del siglo XX el desarrollo de las carreteras estuvo dirigido a complementar la comunicación ferroviaria con caminos que comunicaran a las áreas de producción (Ley Mitre, 1907).

Durante las primeras décadas del siglo XX, la política vial en Mendoza tenía una estructura minúscula. La Dirección de Puentes y Caminos, dependiente del Ministerio de Obras Públicas, había sido creada por la Ley provincial no 393 de 1907, tenía una estructura mínima y su objetivo era mantener la red caminera existente. La Dirección no contaba con reglamentación sobre el trazado de las arterias, regulación y ordenamiento de tránsito, forestación a la vera de los caminos, toma de agua para su conservación. Durante la década de 1920 la agencia estatal encargada de la vialidad se fue profesionalizando y en 1933 se creó la Dirección Provincial de Vialidad, siguiendo el modelo nacional (Sanción de la Ley Nacional de Vialidad y creación de la Dirección Nacional de vialidad - Ley nacional 11.658 de 1932).

En los proyectos y ejecución de las obras intervinieron diferentes agentes, públicos (el Estado provincial, las municipalidades, el Estado nacional, ${ }^{1}$ e instituciones públicas como el Ejército) y semipúblicas (Automóvil Club) que propiciaron el desarrollo de las vías comunicacionales. Asimismo, destaca el papel de los vecinos, quienes en ocasiones

\footnotetext{
${ }^{1}$ En ese tiempo fue muy importante la acción del Estado nacional en relación con la vialidad, aspecto que continuó en el tiempo, ya que muchas obras viales continúan siendo ejecutadas y gestionadas por la Nación.
} 
solicitaron e incluso financiaron obras viales.

Estos daban respuesta a una nueva realidad urbana dada en los años '20 en Argentina debido al crecimiento del parque automotor. El ingreso e incremento de automóviles en la ciudad produjo cambios no sólo en las vías de comunicación, las cuales requirieron ser adaptadas a los requerimientos del tránsito automotor; sino también en la regulación del comportamiento en la vía pública (normativas y ordenamiento del tránsito). Siguiendo a Giucci, podemos señalar que la importancia del automóvil deriva de la centralidad del objeto en la transformación de la vida urbana. ${ }^{2}$ De hecho, la irrupción del automóvil como medio de transporte, generó grandes transformaciones que tuvo como resultado innumerables cambios tanto a nivel sociocultural como en términos político-económicos. ${ }^{3}$

En ese contexto, el objetivo de este artículo es indagar las características de las vías de comunicación y los medios de transporte en Mendoza, centrando el análisis en la agencia estatal encargada de la vialidad provincial. Se analizan ambos aspectos junto con la transformación urbana vinculada a ellos en la década del '20, momento en que la provincia se encontraba gobernada por dirigentes lencinistas (1918-1928). ${ }^{4}$ Para ello se consideran datos estadísticos y proyectos de ley relativos a la vialidad provincial y su recepción en la prensa local a fin de observar su impacto en la opinión pública. Para completar y contextualizar el estudio se alude a las características que estos tenían entre fines del siglo XIX y las primeras décadas del siglo XX.

\footnotetext{
${ }^{2}$ Guillermo Giucci. La vida cultural del automóvil. Rutas de la modernidad cinética (Buenos Aires: Universidad Nacional de Quilmes Editorial/Prometeo, 2007), pp. 16.

${ }^{3}$ Para ampliar información consultar: Valeria Gruschetsky, "Norte - Sur, Este - Oeste. La red de avenidas de la ciudad de Buenos Aires frente al avance de los automotores (1920- 1940)". Estudios del hábitat (en prensa).

${ }^{4}$ La temática abordada fue presentada en forma preliminar como parte de una investigación mayor realizada en la tesis doctoral de la autora. Natalia Luis, La alpargata en el espacio público. Los efectos de la política lencinista en el espacio público mendocino. Tesis de doctorado (Facultad de Filosofía y Letras, Universidad Nacional de Cuyo, Mendoza, Argentina).
} 


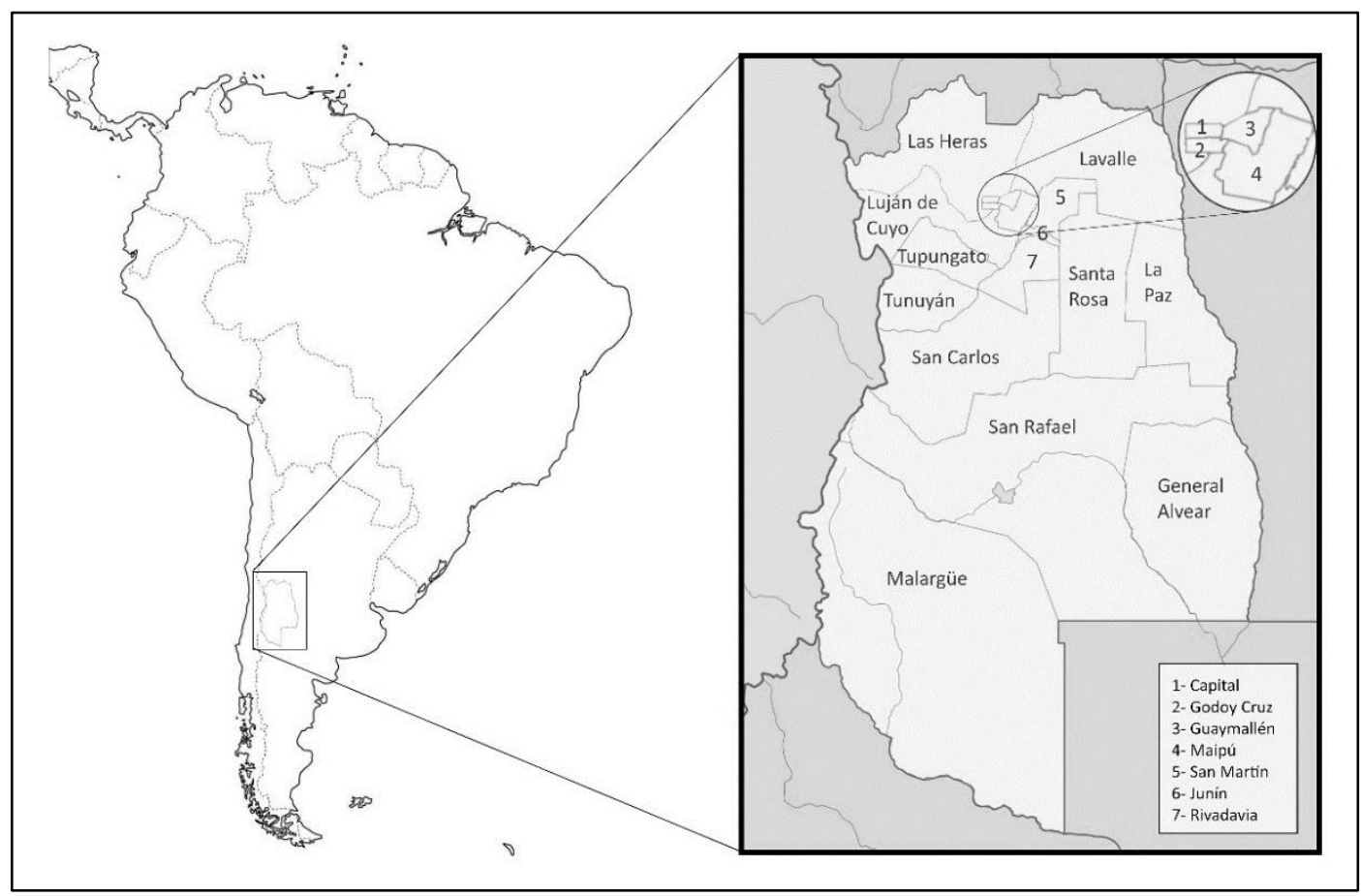

Fuente: elaboración propia

\section{Algunos antecedentes: la historia de la red vial y la formación de burocracia técnica}

Diversos autores han escrito sobre el desarrollo de los medios de transporte y las vías comunicacionales y las transformaciones urbanas que trajeron aparejadas. Entre ellos destaca Giucci, quien analiza el advenimiento de lo que denomina "modernidad cinética", atendiendo al proceso iniciado con la segunda revolución industrial y analizando el surgimiento del automóvil, cómo llegó a convertirse en un elemento de consumo a escala mundial y a ser un símbolo del deporte y la competencia. ${ }^{5}$ Particularmente en Argentina durante la década de 1920 se incrementó de manera relevante el número de automóviles, lo cual trajo aparejado modificaciones urbanas tendientes a integrar este nuevo elemento en el sistema de comunicaciones. Resalta en este sentido la investigación de Ballent y Gorelik, ${ }^{6}$ quienes se refieren al proceso de modernización en el país y explican el papel que cumplió el automovilismo como un fermento del desarrollo industrial, ya que estimuló la industria petrolera para proporcionar combustible y materiales para la construcción de caminos. ${ }^{7}$ Carla

\footnotetext{
${ }^{5}$ Guillermo Giucci, La vida cultural del automóvil...

${ }^{6}$ Anahí Ballent y Adrián Gorelik, "País urbano o país rural: la modernización territorial y su crisis", en: Crisis económica, avance del estado e incertidumbre política tomo VII de la Nueva Historia Argentina, Alejandro Cattaruzza (director de tomo), (Buenos Aires: Sudamericana, 2001), pp. 143-200.

${ }^{7}$ Asimismo, diversos autores aluden al mismo fenómeno en otros países latinoamericanos, entre ellos se puede recordar el aporte de Rodrigo Booth $(2011,2013)$, quien ha analizado en Chile las particularidades del proceso
} 
del Cueto, por su parte, investiga las regulaciones implementadas por los dirigentes para normar el comportamiento del tránsito vehicular a partir de la inserción del automóvil como medio de movilidad. A partir del análisis de las distintas leyes y de los debates parlamentarios muestra cómo las normas se van volviendo más precisas y se ocupan cada vez más de regular aspectos más sutiles. ${ }^{8}$ Además, la autora junto a Gruschetsky estudian puntualmente la implementación del cambio de mano en el tránsito en Argentina en 1945, analizando del marco normativo y su rol en la implementación de una política pública que involucró a todo el territorio nacional, además indagan sobre las transformaciones que generó su implementación - infraestructuras y mecánica- en un contexto expansivo de la vialidad en el continente americano pero atravesado por el contexto de la segunda posguerra y, por último, dan cuenta de las campañas de información y concientización que acompañaron el cambio de mano para analizar las prácticas asociadas a la movilidad vial y sus representaciones. ${ }^{9}$ Por otro lado, Novick, desde un análisis enfocado en los procesos de construcción de la ciudad, examina los debates y las alternativas del proyecto y de la obra de la Avenida 9 de Julio en Buenos Aires, imaginada a fines del siglo XIX y construida en los 30. La autora analiza los diferentes proyectos y ello le permite examinar la conjunción de tiempos cortos y largos, los modos de acción de actores individuales y colectivos, así como las articulaciones entre ideas y cambios en el espacio físico de la ciudad. Asimismo, da cuenta de las lógicas técnicas y las políticas, y revisa el rol que tuvieron las representaciones gráficas como estrategias de propaganda que intervinieron en torno de la obra pública. ${ }^{10}$ Gruschetsky, por su parte, considera la formación del paisaje urbano de la Avenida General Paz como parte de los procesos materiales que transformaron a Buenos Aires en una ciudad moderna durante los años treinta. Señala que el trabajo en conjunto entre ingenieros y arquitectos transformó materialmente el límite de la ciudad, configurando un paisaje definido como "moderno" que combinaba la velocidad que demandaba el automóvil con la comunicación que reclamaba la ciudad. ${ }^{11}$ La autora reflexiona

\footnotetext{
de adopción del automóvil, relacionando la extensión de su uso con el desarrollo de la actividad turística; y a Tomás Errázuriz (2010, 2016), quien reconstruye los hitos fundamentales del proceso de motorización y su impacto sobre la experiencia cotidiana de los viajes en la ciudad de Santiago de Chile en las primeras tres décadas del siglo XX a partir de la incorporación de tres nuevos medios de transporte: el tranvía eléctrico, el automóvil y el autobús.

${ }^{8}$ Carla del Cueto, "Ordenar el tránsito. Leyes y regulaciones de tránsito en la República Argentina 1936-1994", Pasado Abierto. № 8. Julio-Diciembre (2018):212-229.

${ }^{9}$ Carla del Cueto y Valeria Gruschetsky. "Ir por la derecha. Políticas públicas y prácticas de movilidad a propósito del cambio de mano durante el primer peronismo". Pasado Abierto. Revista del CEHis. N¹2. Mar del Plata. Julio-diciembre (2020). ISSN N²451-6961. http://fh.mdp.edu.ar/revistas/index.php/pasadoabierto

${ }^{10}$ Alicia Novick, "La avenida más ancha del mundo. Política, arquitectura e imagen", Anales del Instituto de Arte Americano e Investigaciones Estéticas, 41, (2011): 133-154.to

${ }^{11}$ Valeria Gruschetsky, "El paisaje para un borde urbano: el proyecto para la Avda. Gral. Paz", Anales del Instituto de Arte Americano e Investigaciones Estéticas, n.o 41, (2011): 163.
} 
además cómo y por qué las políticas públicas priorizaron una infraestructura de movilidad que apuntó a favorecer el crecimiento del parque automotor en detrimento de los restantes medios de transporte en Buenos Aires entre 1900 y 1940. ${ }^{12}$ Además, recientemente ha estudiado las intervenciones que conformaron el nuevo sistema de avenidas frente al avance de los automotores, vinculando el diseño vial con el debate urbanístico y analizando la relación técnica y política tanto en la gestión municipal como en la nacional. ${ }^{13}$ Desde otra óptica, Piglia y Pastoriza analizan las vías comunicacionales en relación con la actividad turística. Particularmente aluden a las políticas de turismo social implementadas por el primer peronismo en relación con las obras de infraestructura como caminos, transportes, balnearios y hotelería, en una creciente integración del territorio nacional. ${ }^{14}$

Por otro lado, existen estudios que consideran el papel que cumplieron los agentes públicos y privados en relación con la vialidad. Se puede mencionar los trabajos de Ballent ${ }^{15}$ quien analiza la creación de la Dirección Nacional de Vialidad en 1932 en el marco de un incremento de la relación entre técnica y política. La autora sostiene que la consolidación de la institución marcó un momento culminante y un punto de inflexión en la injerencia de la ingeniería como campo científico y profesional en la estructura estatal de las obras públicas. En la misma línea, Gruschetsky indaga la formación de la burocracia técnica en la DNV, modelo de agencia estatal basado en la "eficiencia" y la "racionalidad", ${ }^{16}$ analizando la circulación de ideas, modelos y experiencias viales que afectaron el desarrollo de esta dependencia, focalizando en los vínculos que se establecieron con los Estados Unidos. La misma autora recientemente ha analizado los vínculos que se establecieron entre el Estado materializado en la DNV-, el conocimiento técnico -encarnado en la ingeniería vial- y la toma de decisiones políticas. ${ }^{17}$ Destaca, además, el aporte de Piglia, quien estudia las intervenciones públicas en materia de vialidad y turismo de los dos clubes de automovilistas

\footnotetext{
${ }^{12}$ Valeria Gruschetsky, "Las nuevas formas de movilidad en la ciudad de Buenos Aires: el avance de los automotores y la política municipal. 1900-1940”, Urbanismo y Transporte Público, editado por Simon Castillo, Marcelo Mardones, y F. Waldo Vila Muga, (Santiago de Chile: RIL Editores, 2018), pp. 48.

${ }^{13}$ Valeria Gruschetsky, "Norte - Sur, Este - Oeste...".

${ }^{14}$ Eliza Pastoriza y Melina Piglia, "La construcción de políticas turísticas orientadas a los sectores medios durante el primer peronismo: Argentina, 1946-1955", Licere, Belo Horizonte, v.20, n.1, (2017):411-452.

${ }^{15}$ Anahi Ballent, "Kilómetro cero: la construcción del universo simbólico en la Argentina de los años treinta", Boletín del Instituto Ravignani 27, núm. 1 (primer semestre 2005): pp. 107-137; y Anahi Ballent, "Ingeniería y Estado: la red nacional de caminos y las obras públicas en la Argentina, 1930-1943", História, Ciências, Saúde Manguinhos, Rio de Janeiro, v.15, n.3, (jul.-set. 2008): p.827-847.

${ }^{16}$ Valeria Gruschetsky, "Saberes sin fronteras. La vialidad norteamericana como modelo de la Dirección Nacional de Vialidad, 1920-1940", en: Los saberes del Estado, coordinado por Plotkin y Zimmermann, (Buenos Aires: Edhasa, 2012), pp. 4.

${ }^{17}$ Gruschetsky, V. (2019). Valeria Gruschetsky, "La Dirección Nacional de Vialidad, una repartición modelo del Estado Nacional. Técnica y política en la Ciudad de Buenos Aires en la década de 1930”, en: Saberes urbanos, L. Menazzi \& G. Jajamovich (Eds.) (Buenos Aires: Teseo, 2019), pp. 77-111.
} 
más importantes de Latinoamérica, el Automóvil Club Argentino y el Touring Club. ${ }^{18}$

Desde la perspectiva de la historia cultural, resalta el aporte de Zunino Singh, quien aborda la movilidad cotidiana en Buenos Aires de principios del siglo XX en tanto práctica y relación social, como un modo más de habitar la ciudad, complementando aspectos ya abordados en los estudios de transporte-como la accesibilidad, uso del tiempo, desigualdad- y ampliando el conocimiento sobre los usos del espacio en los estudios urbanos. ${ }^{19}$ Por otro lado, propone repensar a la ciudad desde sus movilidades a partir de considerar a las prácticas cotidianas de viaje como experiencia urbana. ${ }^{20}$ Además, el autor junto a Giucci y Jirón ${ }^{21}$ han coordinado recientemente un libro que reúne una serie de trabajos sobre conceptos, temas y fenómenos relacionados con las movilidades, entendidas como el movimiento (real, imaginado o potencial) de personas, objetos orgánicos e inorgánicos, dinero, información, recursos. En su análisis, siguen a Sheller y Urry ${ }^{22}$ y a Urry ${ }^{23}$ y entienden el moverse como una forma de habitar. Los artículos del libro forman una especie de diccionario que presenta primero una definición y genealogía del término, y luego un estado del arte que reúne los principales autores o ideas sobre el tema, delineando las principales líneas de investigaciones sobre las movilidades en América Latina. ${ }^{24}$

Si bien existe amplia producción historiográfica latinoamericana y argentina, estas no han estado entre los intereses prioritarios de los historiadores de Mendoza. No obstante, desde la perspectiva histórica tradicional se puede mencionar algunos antecedentes como el trabajo de Santos Martínez, quien ha estudiado los caminos en la época colonial (1561-1810), ${ }^{25}$ y de Lacoste, quien ha analizado las vías de comunicación en relación con los vínculos económicos, políticos y culturales entre Argentina y Chile. Particularmente estudió los

\footnotetext{
${ }^{18}$ Melina Piglia, Autos, rutas y turismo. El automóvil club argentino y el Estado, (Buenos Aires: Siglo XXI, 2014).

${ }^{19}$ Dhan Zunino Singh, "Sea amable, ceda el asiento. Un análisis histórico cultural del comportamiento de los pasajeros en el transporte público de Buenos Aires a principios del siglo XX", Cuaderno Urbano. Espacio, cultura, sociedad, vol. 20, núm. 20, (junio, 2016): pp. 23.

${ }^{20}$ Dhan Zunino Singh, "Ciudades, prácticas y representaciones en movimiento. Notas para un análisis cultural de la movilidad como experiencia urbana", Tempo soc. (online). vol.30, n.2, (2018): 35-54. ISSN 18094554 (Recuperado de: https://www.scielo.br/pdf/ts/v30n2/1809-4554-ts-30-02-35.pdf).

${ }^{21}$ Dhan Zunino Singh, Guillermo Giucci y Paola Jirón, Términos clave para los estudios de movilidad en América Latina, (Ciudad Autónoma de Buenos Aires: Biblos, 2017), 251 pp.

${ }^{22}$ Sheller y Urry, "The new mobilities paradigm", Environment and Planning A volume 38, (2006), pages 207 $226 . \quad$ DOI:10.1068/a37268. Recuperado de: https://www.researchgate.net/publication/23539640 The New Mobilities_Paradigm/link/0deec5260d8b4e8b34 000000/download

${ }^{23}$ John Urry, Mobilities. (Oxford: Polity Press, 2007).

${ }^{24}$ Zunino Singh; Giucci y Jirón, Términos clave..., p. 13-21.

${ }^{25}$ Pedro Santos Martínez, Mapas, planos, croquis y dibujos sobre Cuyo durante el periodo hispánico, (Mendoza: Ediciones Culturales de Mendoza, 1995).
} 
caminos en los Andes Centrales argentino-chilenos (1658-1997), ${ }^{26}$ y el papel que cumplió el ferrocarril Trasandino en el comercio y en la actividad turística de la región. ${ }^{27}$

Además, Ponte ha considerado en sus escritos las vías de comunicación aludiendo, por un lado, a las medidas de ornato y embellecimiento de la ciudad a partir de las primeras calles asfaltadas y cómo estas se fueron ensanchando y extendiendo en la ciudad post terremoto, ${ }^{28}$ y por otro, en relación con la estructuración de los espacios urbanos y rurales a partir de la "cultura del agua". ${ }^{29}$ Según el autor, hay una lógica subyacente en la organización territorial mendocina (tierra de oasis) según la cual la red hídrica era la matriz primaria, y a partir de ella luego se crearon los caminos, que bordeaban los cursos de agua, y finalmente se realizó el catastro adaptado a esa matriz territorial. De esa manera, todo el crecimiento del oasis en el siglo XIX y XX habría seguido esta lógica.

Por último, Luis hizo un acercamiento al estudio de los medios de transporte y las vías comunicacionales en Mendoza entre fines del siglo XIX y las primeras décadas del $\mathrm{XX}^{30} \mathrm{y}$ recientemente ha profundizado el análisis de la expansión del parque automotor en la década del '20. ${ }^{31}$ Asimismo, en un trabajo reciente Raffa y Luis analizaron las transformaciones de la infraestructura vial en Mendoza durante el periodo de gestiones conservadoras (1933-1943). ${ }^{32}$ No obstante, no han sido publicados trabajos que profundicen el estudio de la red vial en la década del '20.

Por otro lado, algunos estudios recientes en Mendoza se dedican a analizar la profesionalización del Estado ${ }^{33}$ Provincial. Entre ellos destacan el aporte de Rodríguez

\footnotetext{
${ }^{26}$ Pablo Lacoste, El sistema pehuenche: frontera, sociedad y caminos en los Andes Centrales argentino-chilenos (1658-1997), (Mendoza: coedición Fac. de Ciencias Políticas y Sociales, Ediciones Culturales y Gobierno de Mendoza, 1998), $230 \mathrm{pp}$.

${ }^{27}$ Pablo Lacoste, El Ferrocarril Trasandino. Un siglo de transporte y política entre Argentina y Chile, (Colección Sociedad y Cultura. Centro de Investigaciones Diego Barros Arana, Santiago de Chile, 2000) 470 pp. ISBN 956-244-114-8; y Pablo Lacoste, El ferrocarril Trasandino y el desarrollo de Los Andes Centrales argentino-chilenos (1872-2013), (Santiago: Editorial Idea, 2013).

${ }^{28}$ Ricardo Ponte, La fragilidad de la memoria. Representaciones, prensa y poder de una ciudad latinoamericana en tiempos del modernismo. Mendoza, 1885/1910, (Mendoza: Fundación CRICYT, 1999), pp. 107-113.

${ }^{29}$ Ricardo Ponte, De los caciques del agua a la Mendoza de las acequias. cinco siglos de la hisloria de acequias. zanjones y molinos, (Mendoza: INCIHUSA-CONICET, 2005), pp. 30-31.

${ }^{30}$ Cfr. Luis, La alpargata ..., cap. IV.

${ }^{31}$ Cfr. Luis, "Automovilismo y transformación urbana. La expansión del parque automotor en Mendoza en la década del '20". En: Carolina Biernat y Nahuel Vassallo, Historia Contemporánea. Problemas, debates y perspectivas (en prensa 2021).

${ }^{32}$ Cecilia Raffa y Natalia Luis, "Caminos para el turismo y la producción. La acción de la Dirección Provincial de Vialidad en Mendoza (1933-1943)", Coordenadas, (7) 2, (2020):146-172.

${ }^{33}$ Es cada vez más diversificada la producción historiográfica sobre los procesos de institucionalización de algunas disciplinas y la relación de los expertos con un sistema internacional que los legitima. Destacan en este sentido a nivel nacional los libros compilados por Mariano Plotkin y Eduardo Zimmerman (2012a y 2012b), quienes abordan desde perspectivas diferentes una serie de procesos históricos en los que se pone de manifiesto el surgimiento de formas específicas de conocimiento y de acciones estatales en áreas tales como la salud, la economía, la defensa, la educación y la seguridad, entre otras. Además, es importante mencionar el trabajo de
} 
Vázquez y Raffa (2016), ${ }^{34}$ de Hirschegger, ${ }^{35}$ de Raffa y Hirschegger; ${ }^{36}$ y los recientes trabajos de Raffa $;{ }^{37}$ y de Barrio y Rodríguez Vázquez ${ }^{38}$ en los cuales se da cuenta de la formación de burocracias técnicas en Mendoza. Además, en los últimos años los investigadores han indagado trayectorias de expertos particularmente relacionados a la actividad vitivinícola durante el período lencinista. ${ }^{39}$ Específicamente sobre la década del '20 Barrio y Rodríguez Vázquez han dado cuenta de cómo el lencinismo mostró la voluntad de institucionalizar las prácticas estatales a través de la incorporación de personal calificado en esas dependencias. ${ }^{40}$ No obstante, resta avanzar en el estudio sobre la profesionalización de la Dirección de Puentes y Caminos y la formación de burocracia técnica vinculada a la red vial en Mendoza en la primera mitad del siglo XX.

Tomando en consideración los aportes previos señalados en relación con las problemáticas que proponen al análisis, en su articulación de infraestructura, dinámica productiva y saberes profesionales, en este artículo se pretende comenzar a explorar y dar

Ernesto Bohoslavsky y Germán Soprano (2010), quienes analizan el Estado y la política a partir de una visión dinámica que presenta una mirada plural de la constitución del Estado y sus funcionarios e instituciones, mostrando su "rostro humano". Resalta del mismo modo el trabajo de Soprano junto a Sabina Frederic y Osvaldo Graciano (2010), en El Estado argentino y las profesiones liberales, académicas y armadas. El volumen se centra en el papel de distintas instituciones formadoras de profesionales relacionando la "génesis, organización, dinámica de las agencias estatales y sociabilidad de sus funcionarios" y los "actores de las instituciones de enseñanza media, terciaria y universitaria" donde se formaron "las profesiones liberales" (2010:13).

${ }^{34}$ El estudio "busca mostrar parte de las respuestas institucionales y las estrategias gubernamentales en relación con la conformación de nuevas agencias estatales y el ingreso de personal técnico a la burocracia de Estado en Mendoza", haciendo referencia no sólo a las tradicionales profesionales liberales sino también a las carreras que tuvieron gran peso en la provincia (tales como la arquitectura y la enología). Cfr: Florencia Rodríguez Vázquez, y Cecilia Raffa, (coords), Profesionalizando un Estado provincial: Mendoza, 1890-1955, (Mendoza: Universidad Nacional de Cuyo-Secretaría de Ciencia, Técnica y Posgrado, 2016), pp. 4.

${ }^{35}$ Ivana Hirschegger, "La Escuela Mixta de Enfermeros de 1942: una apuesta a la profesionalización de la enfermería en Mendoza”, en Trabajos y Comunicaciones, núm. 49, (2019). https://www.doi.org/10.24215/23468971e084

${ }^{36}$ Cecilia Raffa e Ivana Hirschegger, (Comps.), Proyectos y concreciones, obras y políticas públicas durante el primer peronismo en Mendoza (1946-1955), (Mendoza: Universidad Nacional de Cuyo, 2018), 110 pp.

${ }^{37}$ Raffa, Cecilia y Carolina Andrade, Construir Mendoza, (Mendoza, Argentina: Universidad Nacional de Cuyo. Facultad de Filosofía y Letras. Instituto de Historia del Arte, 2020). 1a. edición. 373p. Dirección URL del libro: https://bdigital.uncu.edu.ar/15153.

${ }^{38}$ Patricia Elena Barrio y Florencia Rodríguez Vázquez, Políticas, industrias y servicios en Mendoza (19181943), (Ciudad Autónoma de Buenos Aires: TeseoPress, 2020), Libro digital, EPUB Archivo Digital: descarga y online

ISBN 978-987-86-7673-9.

${ }^{39}$ Cfr.: Florencia Rodríguez Vázquez y Patricia Barrio, "Entre la expertise y la sociabilidad: los aportes de los técnicos extranjeros a una agroindustria regional (Mendoza, Argentina) (1880-1924)", Quinto Sol, vol. 24, no 2, (mayo-agosto 2020), ISSN 1851-2879, pp. 1-19. DOI: http://dx.doi.org/10.19137/qs. v24i2.4054; y R. A. Abraham, "Un abogado al frente de la \&quot;policía del vino", Estudios Sociales Contemporáneos, (21), (2019), pp. 19-39. Recuperado a partir a de https://revistas.uncu.edu.ar/ojs3/index.php/estudiosocontemp/article/view/2640

${ }^{40}$ Patricia Barrio y Florencia Rodríguez Vázquez, "Instituciones, saberes y expertos para un Estado provincial: Leopoldo Suárez, de agrónomo a funcionario itinerante del lencinismo, Mendoza, 1918-1930", Boletín del Instituto de Historia Argentina y Americana "Dr. Emilio Ravignani”, Tercera serie, núm. 44, (primer semestre 2016), pp. 78-107. 
cuenta de las particularidades de la provincia de Mendoza en materia de vialidad en la década de 1920.

Se postula como hipótesis que, en el marco de una creciente profesionalización de las instituciones estatales a comienzos del siglo XX, los dirigentes lencinistas acudieron de un modo creciente a personal capacitado para los proyectos y ejecución de obras viales, incorporándolos a las instituciones públicas. Así se habría incrementado la profesionalización de la Dirección de Puentes y Caminos. Los gobernantes plantearon crear nuevas vías de comunicación y ampliar las existentes a fin de conectar lugares e integrar zonas periféricas, favoreciendo una mayor integración territorial a nivel provincial, considerada como un símbolo del progreso. Del mismo modo, plantearon extender el servicio de transporte para facilitar el arribo de los sectores de clase media y popular a lugares antes inaccesibles, propiciando con ello la democratización del servicio.

\section{El periodo de estudio}

El arco temporal que se indaga está enmarcado en un contexto signado por la ampliación de la esfera pública. Luego de un largo periodo de orden conservador caracterizado por el fraude electoral, entre fines del siglo XIX y comienzos del XX nuevas olas de radicalización transformaron las prácticas políticas en Latinoamérica. En Argentina nació de la crisis de 1890 y tomó cuerpo con la Unión Cívica Radical (UCR) bajo el liderazgo de Hipólito Yrigoyen. En ese contexto fue particularmente importante la Ley Sáenz Peña (1912), que implicó un cambio significativo al instaurar la obligatoriedad y el secreto del voto y la representación de la minoría permitiendo elecciones más transparentes que trajeron aparejado un proceso de democratización política. ${ }^{41}$ Las elecciones de 1916 llevaron al gobierno nacional a la UCR y dos años después salió triunfante el radicalismo lencinista en Mendoza.

José Néstor Lencinas y luego su hijo Carlos Washington, lideraron lo que algunos autores llaman el populismo mendocin. ${ }^{42}$ Estos gobiernos esgrimieron un discurso

\footnotetext{
${ }^{41}$ Para ampliar información consultar: Persello, Ana Virginia Persello, Historia del radicalismo, (Buenos Aires: Edhasa, 2007); Waldo Ansaldi, "La trunca transición del régimen oligárquico al régimen democrático", en: Nueva Historia Argentina, Tomo VI, Falcón, Ricardo (dir.), (Buenos Aires: Sudamericana, 2000); y, Hilda Sabato, Marcela Ternavasio, Luciano De Privitiello y Virginia Persello, Historia de las elecciones en la Argentina, (Buenos Aires: El Ateneo, 2011).

${ }^{42}$ Los autores caracterizan al lencinismo como un caso de populismo cuyano, junto al cantonismo de San Juan. Para ampliar información consultar: Richard Jorba, "Los gobiernos radicales de los Lencinas en Mendoza"; Richard Jorba, "Los orígenes del fenómeno populista en Mendoza"; Rodríguez, Lencinas y Cantoni; Lacoste, La Unión Cívica Radical en Mendoza y en la Argentina.
} 
antioligárquico que se tradujo en políticas públicas propuestas e implementadas destinadas a mejorar la calidad de vida de la población, que incluyeron participación para sectores de clase media y baja en ámbitos que antes eran exclusividad de élites. ${ }^{43}$

El periodo fue inestable. Hubo 3 gobernaciones y 4 intervenciones federales, ${ }^{44}$ lo que generó una escasa continuidad de las propuestas políticas que se encontraban en marcha. Ello, sumado a la inestabilidad económica ${ }^{45}$ (durante el periodo se alternó una época de auge, a la cual siguió una importante crisis financiera) hizo que muchos de los planes ideados no pudieran concretarse o se retrasara su ejecución.

Es importante mencionar, además, que entre fines del siglo XIX y principios del XX comenzó en América Latina y Argentina un proceso de profesionalización de las dependencias estatales, por la cual las instituciones fueron acudiendo más asiduamente a personal técnico para cubrir los puestos. A su vez, esas mismas reparticiones se convirtieron en ámbitos de formación y fueron incrementando el cuerpo técnico estatal ${ }^{46}$. También durante ese lapso temporal se reglamentó la práctica profesional. Específicamente en Mendoza se normó de manera más rigurosa la medicina, obstetricia y farmacia en 1927, y hubo un proyecto de ley que proponía reglamentar las carreras de arquitectura, ingeniería y agrimensura en 1922, que, si bien no se sancionó, da cuenta del avance estatal en la reglamentación del ejercicio profesional.

\section{La Dirección de Puentes y Caminos: una naciente burocracia técnica}

Como se explicó en páginas anteriores, durante los primeros años del siglo XX el equipamiento institucional y legal en materia vial en Mendoza recién se estaba gestando. La Dirección de Puentes y Caminos, dependiente del Ministerio de Obras Públicas había sido creada por ley 393 de 1907 y no existía un plan sistemático de desarrollo en relación con las

\footnotetext{
${ }^{43}$ Para ampliar información consultar: Luis, La alpargata...

${ }^{44} \mathrm{Si}$ bien Lencinas había liderado la revolución radical de 1905 en Mendoza, apenas asumió el poder como gobernador se produjo una división entre radicales y lencinistas (Lacoste, La Unión Cívica Radical..., pp. 72), aspecto que influyó para que las dirigencias provinciales fueran interrumpidas mediante intervención federal.

${ }^{45}$ Para ampliar información consultar: Patricia Barrio, "Regulación e intervención estatal en tiempos turbulentos. El caso de la vitivinicultura mendocina entre 1918 y 1923”, en: De la regulación a la intervención en tiempos de cambio. Las prácticas y vínculos estatales en la provincia de Mendoza, 1916-1970, Florencia Rodríguez Vázquez (et.al.), (Rosario: Prohistoria Ediciones, 2014); Patricia Barrio, "Un proyecto efímero de regulación privada de la vitivinicultura en la provincia de Mendoza (República Argentina) en contexto de crisis: la Sociedad Vitivinícola de Mendoza, 1916-1919”, en: Revista Historia y Sociedad, Facultad de Ciencias Humanas y Económicas de la Universidad Nacional de Colombia, Bogotá, (2015):167-197, y Rodolfo Richard Jorba, "Los frutos del viñedo deberían ser para todos. Depresión y resurrección de la vitivinicultura y aumento de la conflictividad social en Mendoza (Argentina), 1919-1920”, en: Estudios Sociales, 45, Rosario, (2013): 71-101.

${ }^{46}$ Cfr: Anahi Ballent, "Kilómetro cero..."; Anahi Ballent, "Ingeniería y Estado...”; y ${ }^{46}$ Valeria Gruschetsky, "Saberes sin fronteras...".
} 
vías de comunicación. Durante el periodo de gobernaciones lencinistas se fue dotando de recursos económicos, lo cual permitió la creación de nuevos puestos de personal técnico que comenzaron a formar parte de la burocracia del estado provincial. Por ejemplo, en abril de 1918 se propuso crear el puesto de "dibujante", al cual le correspondería un sueldo mensual de $\$ 200 .^{47}$ Además, en septiembre del mismo año, la cámara de senadores aprobó un proyecto de ley del Poder Ejecutivo que creaba un impuesto a los terrenos cultivables e incultos, para reforzar los fondos de la ley 393 sobre conservación de Puentes y Caminos. ${ }^{48}$

$\mathrm{Al}$ año siguiente se legisló en el mismo sentido para obtener más recursos para atender a la infraestructura vial. El proyecto de ley estaba firmado por Leopoldo Suárez, ${ }^{49}$ que ocupaba el cargo máximo del ministerio de Obras Públicas de la provincia. En la sesión legislativa, el senador Egidio Casnati aludía a la importancia de conservar las vías comunicacionales para el desarrollo de la economía provincial. Reflexionaba: "Nadie ignora la necesidad que se tiene de estos caminos para el transporte de los productos de la Provincia, de uno a otro Departamento, de una a otra región. No sería, pues, una economía, permitir que esos caminos se destruyan". ${ }^{50} \mathrm{El}$ proyecto se sancionó y se incrementó el dinero disponible para la conservación de los caminos provinciales.

Durante el período de gestiones lencinistas se incorporó la consideración del regadío del arbolado público vinculado a los caminos con la antigua Ley de Aguas promulgada en 1815. Por intermedio de esa enmienda, los inspectores de canales, hijuelas y demás cauces de riego en todo el territorio de la provincia estaban obligados, a requerimiento de las autoridades de la Sección de Puentes y Caminos, a proporcionar a los camineros o cuidadores de la conservación de las vías públicas el agua del o de los cauces que mejor conviniere para el riego de aquéllas y de sus arbolados por el tiempo necesario a tal objeto. Si bien no se analiza con detenimiento esta variable, cabe mencionar que la vinculación entre camino y árbol marca una de las particularidades de la vialidad en Mendoza, hasta la actualidad. ${ }^{51}$

Tanto en el nivel nacional como en el provincial había inspectores de la Dirección de

\footnotetext{
${ }^{47}$ Diario de sesiones legislativas de la Cámara de senadores. Sesión correspondiente al día 26 de julio de 1918.

${ }^{48}$ El mismo era un impuesto para incrementar los recursos disponibles para la conservación de los caminos de la provincia y regiría a partir del 1 de enero de 1919. Si bien este proyecto fue propuesto por el gobernador anterior (Francisco Álvarez), fue sancionado durante la gestión lencinista.

${ }^{49}$ Leopoldo Suárez fue un enólogo e ingeniero agrónomo, formado en la provincia y perfeccionado en el extranjero que desempeñó numerosos cargos públicos en Mendoza. Fue superintendente General de Irrigación (julio 1918-mayo de 1919), luego ministro de Obras Públicas entre julio 1919 y enero 1920; y posteriormente entre enero1922 y octubre 1924. Además, ejerció como interventor de la Dirección General de Escuelas entre agosto de1926 y abril de 1927. Cfr: Barrio y Rodríguez, "Leopoldo Suárez...”, p. 104.

${ }^{50}$ Senador Casnati. Diario de sesiones legislativas de la Cámara de senadores, sesión correspondiente al día 10 de octubre de 1919.

${ }^{51}$ Raffa y Luis, "Caminos para el turismo y la producción..."
} 
Puentes y Caminos cuya tarea era realizar viajes a los diferentes lugares a fin de constatar el estado de la red vial. También tenía la misión de multar a los regantes: uno de los principales factores que deterioraban significativamente el estado de la calzada en Mendoza eran los desbordes de las acequias de riego que humedecían el suelo y al paso de los vehículos dejaban profundas improntas que luego de secarse dificultaban la circulación posterior. La prensa daba cuenta de dichas intervenciones. ${ }^{52}$

Con respecto a la organización de la dependencia, se sabe que en 1914 la Dirección de Puentes y Caminos estaba dividida en 7 secciones. A comienzos de 1918 se fraccionó en 9, llevándose registrado y controlado el movimiento de estas en la oficina central. El número de secciones fue variando conforme pasaba el tiempo. ${ }^{53}$ En general se conformaron por grupos de departamentos, por departamento, y más contemporáneamente también se incluyeron distritos importantes en material vial como Potrerillos, sitio turístico de la región oeste de Mendoza.

Además, a partir de 1925 se comenzó a contabilizar la cantidad de kilómetros construidos en los departamentos, permitiendo registrar de manera fehaciente la infraestructura vial de la provincia (ver Ilustración 3). Así, la información registrada a partir de los datos estadísticos servía de base para planificar las políticas públicas

De esa manera, durante la década de 1920 la repartición de Puentes y Caminos fue incorporando recursos financieros y humanos y se fue profesionalizando. Contaba, entre su personal, con técnicos y especialistas encargados de inspeccionar el estado de las carreteras provinciales y de proyectar nuevas obras viales que cumplieran las normas de seguridad antisísmicas necesarias para la región de cuyo. La ingeniería se había convertido en una profesión clave al servicio del Estado provincial. Ello, sumado a los datos que arrojaba el conocimiento estadístico sirvió de base para la planificación vial. Además, a lo largo de las primeras décadas del siglo XX el desarrollo de nuevas tecnologías para la construcción de caminos y puentes avanzó notablemente. Nuevos materiales y sistemas produjeron avances significativos en la construcción y reparación de las vías de comunicación (adoquinados, estabilizados (macadam), pavimentos (asfalto), entre otros).

\footnotetext{
${ }^{52}$ En 1927 se comunicaba que el jefe de la cuarta sección de Puentes y Caminos de la Nación había realizado un viaje por todo el sur de la provincia, acompañado por dos ingenieros y un empleado de dicha repartición a fin de observar el estado de los caminos y la necesidad de realizar nuevas obras de vialidad en el sur (Los Andes, 25/10/1927).

${ }^{53}$ A partir de 1934, cada uno de los departamentos de la provincia representó una sección distinta.
} 


\section{Los medios de transporte en Mendoza entre fines del siglo XIX y las primeras décadas del siglo $\mathrm{XX}^{54}$}

A fines del siglo XIX (1885) llegó el ferrocarril a la provincia, trayendo aparejado no solo el incremento del acceso de la producción mendocina al mercado nacional e internacional, sino posibilitando también el flujo de personas e ideas con mayor dinamismo. Del mismo modo, fueron apareciendo a lo largo del periodo otros medios de transporte. A los cocheros que conducían carruajes de tracción a sangre se sumó el tranvía eléctrico a principios del siglo $\mathrm{XX}^{55}$, y en forma paulatina, los automotores, que fueron propagándose en la ciudad y luego en los departamentos de la campaña.

Sumado a la red tranviaria, a partir de 1920 funcionó el servicio de autobús, que facilitó igualmente el traslado de los sectores de clase media y baja. Dos años después la provincia ya contaba con 17 líneas de ómnibus que prestaban servicio entre la capital y los departamentos (ver ilustración 2). El incremento que alcanzó el servicio en la provincia hizo necesario que se reglamentara la prestación para garantizar la seguridad pública, ya que se habían originado trastornos por la mala conducción de los vehículos, por la deficiente construcción de los coches y por la trepidación de estos con excesivo peso. Una de las medidas implementadas consistió en corroborar la competencia de los conductores. Así, en la Municipalidad se abrió un registro para las personas que manejaban ómnibus y autobuses, quienes comenzaron a ser sometidos a un examen técnico de competencia ${ }^{56}$.

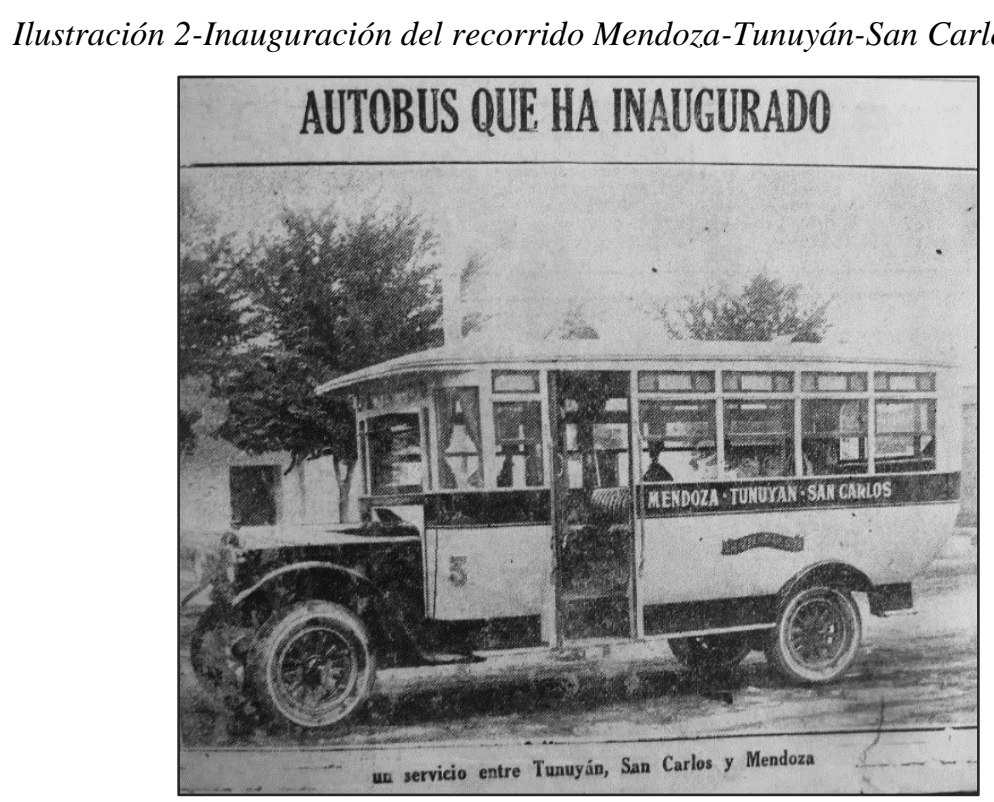

Fuente: La Palabra, 2 de enero de 1928

\footnotetext{
${ }^{54}$ Una versión preliminar de este apartado fue presentada en las Jornadas de Historia Moderna y Contemporánea, 2021, en prensa.

${ }^{55}$ El 1 de octubre de 1912 comenzó a circular el tranvía en Mendoza.

${ }^{56}$ La memoria del siglo, Los Andes, (2000), pp. 499, 504-506.
} 
A fines de 1928 se sumó un nuevo medio de transporte público, el taxi-colectivo. El servicio era prestado por la empresa Taxibus Mendoza, que poseía 20 vehículos. Estos trasportaban 6 o 7 personas.

Al incremento de vehículos dedicados al transporte público, se sumó la expansión de los automotores de uso particular. En 1914, la Dirección General de Estadísticas contabilizó 18.106 vehículos matriculados, ${ }^{57}$ de los cuales 286 eran automóviles y 30 motocicletas, lo cual arroja un total de 316 vehículos motorizados. ${ }^{58}$ Ahora bien, del total de automóviles, 150 (es decir un número muy significativo del total) estaban radicados en capital, y el resto en los departamentos. En 1922 ya había 1582 automóviles en la provincia. Este número creció de manera significativa llegando a 5.511 automóviles en 1926 (esto correspondía proporcionalmente a un coche cada 67 habitantes ${ }^{59}$ ). El incremento de vehículos motorizados se verificaba en todo el país y en los países industrializados. ${ }^{60}$ A fines de 1928, Mendoza contaba con 9.445 automotores y se consumían 17.705, 066 litros de nafta. ${ }^{61}$

Todo ello trajo aparejado el desarrollo de la industria y comercio de combustible. En 1916 se consumía aproximadamente 1000 litros, en 1925 más de 8000, y en 1928 se llegó a 17705,066 litros. Más allá del consumo, Mendoza comenzó a producir combustible para atender la demanda local ${ }^{62}$. A partir de 1920 se empezó a destilar nafta en las instalaciones de Ramón Bennet, en Luján, y en 1922 se instalaron bombas para el expendio. En 1926 la nafta vendida era elaborada por Yacimientos Petrolíferos Fiscales (YPF), siendo el primer concesionario Posca Hermanos y Compañía. ${ }^{63}$

Por otro lado, el incremento del número de automóviles generó nuevas problemáticas urbanas tales como el acrecentamiento del ruido y el aumento de los accidentes de tránsito. Mediante la prensa se solicitaba que los dirigentes provinciales intervinieran de manera más sistemática a fin de garantizar la seguridad vial. Específicamente se referían a la necesidad de que las autoridades normaran y controlaran el comportamiento vehicular en las calles. Asimismo, aparecían notas ofreciendo consejos a los automovilistas sobre el buen manejo de

\footnotetext{
${ }^{57}$ Se contabilizaban además coches, carruajes, carreteras, carros tirados por bueyes, entre otros.

${ }^{58}$ Anuario de la Dirección general de estadísticas correspondiente al año 1914. 1916. Buenos Aires: Talleres S. A. Casa Jacobo Peuser, LTDA

${ }^{59}$ La Memoria del siglo..., pp. 507.

${ }^{60}$ En 1927 en Argentina se contabilizaba un coche cada 49 habitantes, en Francia cada 43, en Gran Bretaña cada 39 y en EE. UU. cada 6. Argentina llegó a ser en la entreguerra, el país latinoamericano con más automóviles y osciló entre el cuarto y séptimo lugar mundial. Tenía un número muy elevado de automóviles en relación con su población y con su escaso kilometraje de caminos permanentes. Cfr: Melina Piglia, "Turismo en automóvil en Argentina", Tempo Social, revista de sociologia da USP, v. 30, n. 2, (2018): pp. 87-88.

${ }^{61}$ Anuario de la Dirección general de estadísticas correspondiente a los años 1927-1929. 1931. Buenos Aires:

Talleres S. A. Casa Jacobo Peuser, LTDA.

${ }^{62}$ Luego, con el incrementó en la producción de nafta esta también se comercializó en el exterior.

${ }^{63}$ La Memoria del siglo..., pp. 380, 432-439, y 499.
} 
los vehículos a los fines de acompañar los esfuerzos gubernamentales. Si bien en $1913^{64}$ se había dictado un decreto con ciertas regulaciones, como la que estipulaba que los automóviles debían circular a un máximo de $14 \mathrm{~km} /$ hora en la ciudad y a $25 \mathrm{~km} / \mathrm{hora}$ en los barrios urbanos de la campaña, en 1926 se reglamentó de manera más exhaustiva la conducción. Se establecieron horarios de carga y descarga de mercadería, estacionamientos prohibidos y se reglamentó la circulación por una sola mano de ciertas calles céntricas. No obstante, los accidentes de tránsito se seguían produciendo con mucha frecuencia. ${ }^{65}$

Así, desde fines del siglo XIX y de manera particular en la década del veinte aparecieron y se extendieron nuevos medios de transporte en Mendoza, que generaron no solo la necesidad de ampliar, mejorar y complejizar la infraestructura vial existente, sino comenzar a normar comportamientos públicos nuevos para garantizar la convivencia. A su vez, esto requirió la especialización de ciertos saberes, los cuales sirvieron para que el Estado profundizara su intervención efectiva. Asimismo, el incremento de medios de transporte público y la ampliación de los recorridos facilitó el traslado de los sectores de clase media y popular a lugares antes inaccesibles. ${ }^{66}$

\section{Las vías comunicacionales en la década del '20. Del discurso a las obras}

El discurso de los dirigentes lencinistas giró en torno a la necesidad de construir nuevos caminos y conservar los existentes para conectar la provincia, facilitando el flujo de personas y producción en las zonas retiradas. Estos postulaban la mejora en la red vial como un símbolo del progreso provincial. Tanto José Néstor Lencinas como Alejandro Orfila aludieron al progreso que proporcionaría la mejora de la red de caminos. José Néstor Lencinas había prometido con anterioridad a su elección como gobernador, que desarrollaría la vialidad pública para que se facilitara el transporte de los productos: "Mendoza debe completar su red de caminos provinciales, que con los que tiene a su cargo la Nación por la ley Mitre, sea un exponente de nuestro progreso". 67

En 1927 Orfila señalaba: "convencido de que los gobiernos surgidos de una lid democrática, como expresión de las aspiraciones públicas deben trabajar incesantemente por

\footnotetext{
${ }^{64}$ El 13 de enero de 1913 el gobernador de Mendoza dictó un decreto reglamentando el tráfico y movimiento de caminos de la provincia. El mismo había sido formulado por una comisión nombrada a tal efecto el año anterior. Cfr: Villegas, Norberto Amancio Villegas, Cien años de nuestra vialidad. Mendoza, (2003): 44-46.

${ }_{65}^{65}$ La Memoria del siglo...pp. 499-503.

${ }^{66}$ Para ampliar información consultar: Natalia Luis, "Automovilismo y transformación urbana. La expansión del parque automotor en Mendoza en la década del '20" (en prensa).

${ }^{67}$ Julio Nieto Riesco, José Néstor Lencinas. Jefe de Estado, (Mendoza: Talleres Gráficos Argentinos I.J. Rosso, 1926), p. 255
} 
el progreso de la provincia para evidenciar de ese modo que han logrado responder a la confianza de la opinión, he dedicado preferente atención a la mejora del régimen caminero de Mendoza" (...) "Anhelo que mi paso por el gobierno, sea señalado con una serie de obras públicas, que, como la construcción de puentes, desagües, canales, diques, y casas departamentales, respondan a la necesidad de rodear de ventajas y beneficios a toda la población". 68

En cuanto a las obras de infraestructuras, se realizaron especialmente en las áreas de mayor densidad. En 1925 existían 1.387 Km. y 200 mts. de caminos en la provincia, sin considerar la capital provincial. Los departamentos cercanos a la capital y que tenían una alta tasa de densidad poblacional, como Guaymallén y la zona de Godoy Cruz y Luján, contaban con una significativa cantidad de kilómetros de caminos construidos (ver ilustración 3). La excepción era el departamento de Las Heras, se trataba de un espacio marginado de los proyectos de modernización y por ello desjerarquizado, ${ }^{69}$ y se hallaba fuera de los centros agro-productores, no era uno de los principales departamentos que producía artículos comercializables en los periodos de cosecha.

Ilustración 3- Extensión total de caminos sostenidos por la provincia. Mendoza, 1925-1927

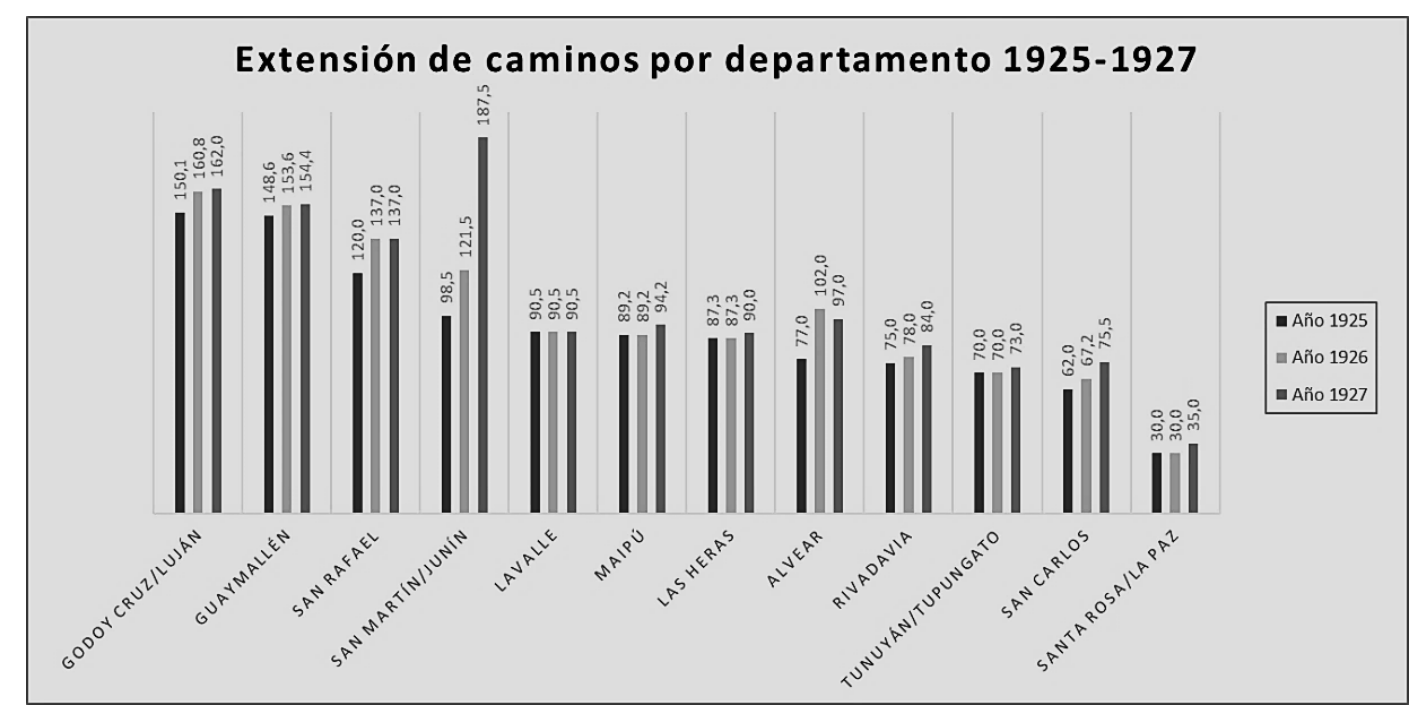

Fuente: elaboración propia en base a los Anuarios estadísticos correspondiente a los años 1925-1927

Es importante marcar que, si bien durante la década del '20 fueron numerosas las propuestas de creación de nuevos caminos o de finalización de algunas obras ya comenzadas, estas se vieron frenadas y algunas no realizadas debido en gran parte a las intervenciones

\footnotetext{
${ }^{68}$ Mensaje inaugurando el periodo legislativo 1927. En: Los Andes, 7/6/1927.

${ }^{69}$ Ponte, La fragilidad..., p 455.
} 
federales, tal como ocurrió con otras obras de infraestructura. ${ }^{70}$ En efecto, dirigentes del gobierno interventor en ocasiones dejaban sin efecto medidas ya implementadas, se paralizaban algunos trabajos y se optaba por priorizar obras de distinta índole.

Durante la gestión gubernamental de Alejandro Orfila y persiguiendo su ideal de ser reconocido en la posteridad por las obras públicas (y entre ellas las obras viales), se extendieron los caminos de la provincia, llegando a tener $1.564 \mathrm{~km}, 318 \mathrm{mts}$ de extensión total en diciembre de $1927 .{ }^{71} \mathrm{De}$ los $1280 \mathrm{~km}$ de caminos sostenidos por el gobierno provincial, $921 \mathrm{~km}$ 500mts estaban sistematizados (consolidados) en tierra y $358 \mathrm{~km}$ con 600 mts se encontraban enripiados (ver ilustración 4). Cabe destacar que los departamentos de San Martín y Junín integraron la zona en la que más creció la red vial durante la última gestión lencinista. $^{72}$

Ilustración 4- Cuadro. Extensión de los caminos sostenidos por la provincia por sistema

\begin{tabular}{|l|c|c|c|}
\hline \multirow{2}{*}{ Sistema constructivo } & \multicolumn{2}{c|}{ Extensión por año [km] } \\
\cline { 2 - 4 } & 1925 & 1926 & 1927 \\
\hline Macadamizado & 151,6 & & \\
Sistematizado en tierra & 521,2 & 503,0 & 921,5 \\
Enripiado & 164,6 & 354,1 & 358,6 \\
Medio enripiado & 64,8 & & \\
Conservado para el tráfico con cunetas & 196,0 & & \\
Conservación extraordinaria & & 330,0 & \\
\hline Total & 1098,2 & 1187,1 & 1280,1 \\
\hline
\end{tabular}

Fuente: elaboración propia en base a los datos estadísticos de 1925-1927

Como es posible observar en el cuadro anterior, en la década del '20 se utilizaba principalmente el sistema enripiado y el sistematizado en tierra para la conservación de caminos sostenidos por la provincia. En 1925 cerca del 50\% (el 47, 45\%) de los caminos estaban sistematizados, y casi un 15\% enripiados. Para 1927 aproximadamente el $72 \%$ del total de caminos estaban sistematizados en tierra y el $28 \%$ se encontraba enripiado. Esto se modificó en la década posterior a partir de la radicación de dos fábricas de cemento portland: MINETTI y CORCEMAR, en el departamento de Las Heras, Mendoza. Amparadas en las Leyes $\mathrm{N}^{\circ}$ 978/32 y 1.065/33. La posibilidad de contar con producción local modificó el costo del material, que se hizo más económico, y facilitó su utilización transformando el panorama

\footnotetext{
${ }^{70}$ Para ampliar información consultar: Luis, La alpargata ..., pp. 277-284.

${ }^{71}$ Anuario de la Dirección general de estadísticas correspondiente a los años 1927-1929. 1931. Buenos Aires: Talleres S. A. Casa Jacobo Peuser, LTDA.

${ }^{72}$ Los datos estadísticos de 1928 sobre la red vial no se encuentran en el Anuario, esto probablemente se deba a que en octubre de 1928 la provincia fue intervenida.
} 
local vinculado a la obra pública: el hormigón de cemento portland, se convirtió en el elemento incuestionable para construir pavimentos en la región andina. ${ }^{73}$

Además de las iniciativas provinciales, el Estado nacional tuvo un papel importante en la ejecución de obras viales. En 1926 los periódicos anunciaban que el gobierno nacional planeaba diversas obras de vialidad en Mendoza, tanto interdepartamentales como caminos de unión entre provincias e incluso con el vecino país trascordillerano. Se programaba, entre otros, la ejecución de un tramo del camino internacional a Chile, el camino de Mendoza a San Rafael, y el camino de Mendoza a San Juan (particularmente el tramo correspondiente a Mendoza). ${ }^{74}$

Otra labor vial proyectada por la Nación fue la reparación del camino a las termas de Villavicencio. ${ }^{75}$ En el mismo sentido, a fin de desarrollar el turismo provincial, el gobernador Alejandro Orfila propuso acelerar los trabajos del camino a Cacheuta contratando más personal. El mismo sería inaugurado el 28 de enero del año siguiente. La obra fue inaugurada el 28 de enero de 1928. El diario La Palabra señalaba que el puente que cruza sobre el Río Mendoza constituye "una meritoria obra de ingeniería, pues se trata del puente mayor que existe en Sud América construido de cemento armado, siendo su extensión total de 45 metros, construido de un solo arco, que en su base es de 30 metros" ${ }^{, 76}$ (Ver ilustración 4). De esa manera, la prensa daba cuenta de la utilización de cemento armado y demás materiales resistentes en la obra pública. ${ }^{77}$

\footnotetext{
${ }^{73}$ Boletín de Obra Pública de la República Argentina. (1938). Buenos Aires, Argentina: Ministerio de Obras Públicas de la Nación, pp. 421. Para ampliar información cfr: Raffa y Luis, Caminos para el..., p.157.

${ }^{74}$ Nota periodística: Los Andes, 8/10/1926.

${ }^{75}$ Además, la Nación aprobó en 1928 la partida de dinero correspondiente para la construcción de un puente en Barrancas, del departamento de Maipú. El mismo se construyó con posterioridad a las gobernaciones lencinistas. ${ }^{76}$ Nota periodística de: La Palabra, 30 de enero de 1928.

${ }^{77}$ Para ampliar información sobre la utilización de nuevos materiales y sobre la construcción sismorresistente en Mendoza consultar: Natalia Luis y Matías Esteves, "Técnica y política en la construcción sismorresistente en Mendoza en la década del '20. La acción del Estado en los casos de Costa de Araujo y Villa Atuel”, en: Estudios sociales del Estado, (en prensa).
} 


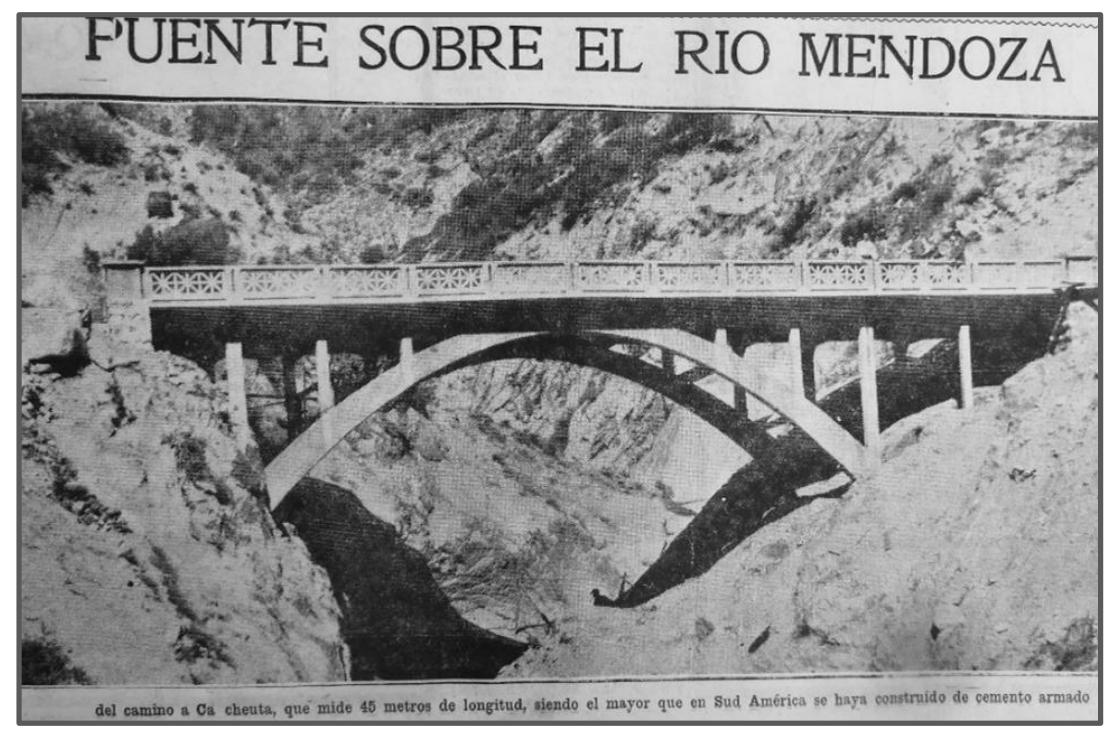

Fuente: La Palabra, 30 de enero de 1928

El Ejército también asumió un papel significativo en la vialidad provincial. Con motivo de los ejercicios militares, se procedía a mejorar y/o construir nuevas carreteras y puentes. ${ }^{78}$ La prensa publicada durante estos años daba cuenta de los trabajos efectuados. Por otro lado, es importante marcar que, a diferencia de lo acaecido en otras provincias, en Mendoza el Automóvil Club Argentino (ACA) no tuvo mucha incidencia en la expansión del parque automotor y en la ejecución de obras viales durante la década de $1920 .{ }^{79}$

Asimismo, es importante mencionar que hubo una articulación constante entre la entidad provincial encargada de vialidad, la Dirección de Puentes y Caminos, y las autoridades municipales de los diferentes departamentos. Al respecto, Los Andes señalaba con respecto a la vialidad: "Es preciso, entonces, que todas las reparticiones del Estado que algo tienen que ver con el asunto, trabajen de conjunto para lograr la mayor suma de eficacia y éxito en la tarea". ${ }^{80}$

En relación con los departamentos, durante el periodo se repararon varías vías de unión interdepartamentales y se construyeron otros puentes. Tal fue el caso del puente carretero sobre el río Atuel (ver ilustración 6). Carlos W. Lencinas sancionó un decreto y se construyó en 1922 en la antigua ruta 143, camino a la estación de Negro Quemado,

\footnotetext{
${ }^{78}$ Los puentes y caminos construidos por el Ejército estaban destinados principalmente a facilitar el acceso a los regimientos, pues los fondos provenían en general del Ministerio de Guerra.

${ }^{79}$ el Automóvil Club Argentino recién contó con sede en la provincia en 1924. Particularmente se instaló el 13 de agosto de 1924 en la calle Lavalle 108. Dos años después, en Mendoza había 80 socios, mientras que, en la vecina provincia de San Juan, ya llegaban a 289. Para ampliar información Cfr: Luis, la alpargata..., pp. 212266; y Luis, “Automovilismo y transformación urbana...".

${ }^{80}$ Los Andes, 7/01/1924.
} 
facilitando entonces la comunicación con el departamento de San Rafael. La iniciativa había sido de los vecinos de la zona, y el Poder Ejecutivo se unió y apoyó económicamente gran parte del monto total de la obra.

Ilustración 6- Foto Inauguración puente carretero sobre el río Atuel

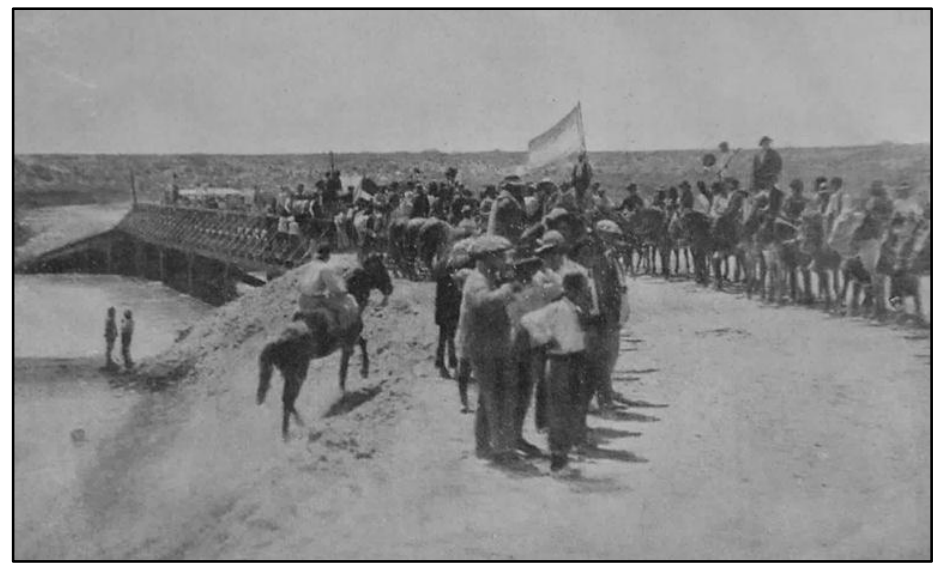

Fuente: Memoria del Ministerio de Obras Públicas. Ingeniero Leopoldo Suárez, 1922-1923

Por otro lado, a fin de facilitar la comunicación entre la capital provincial y el departamento de Guaymallén, fue construido un puente desde la calle Morón sobre el canalzanjón (ver Ilustración 6). El mismo fue construido principalmente por fondos otorgados por una vecina, quien donó también terreno para la apertura de la calle Morón desde el puente hasta la calle Dorrego. La obra se inauguró el 11 de septiembre de 1928. Los Andes señalaba: "Este puente, que viene a unir a la ciudad con Guaymallén, abre una nueva arteria que facilitará el tráfico con este departamento, favoreciendo a un barrio que, por su proximidad a nuestro centro, está llamado a adquirir inmediata importancia", 81

Ilustración 7- Foto Puente sobre calle Morón

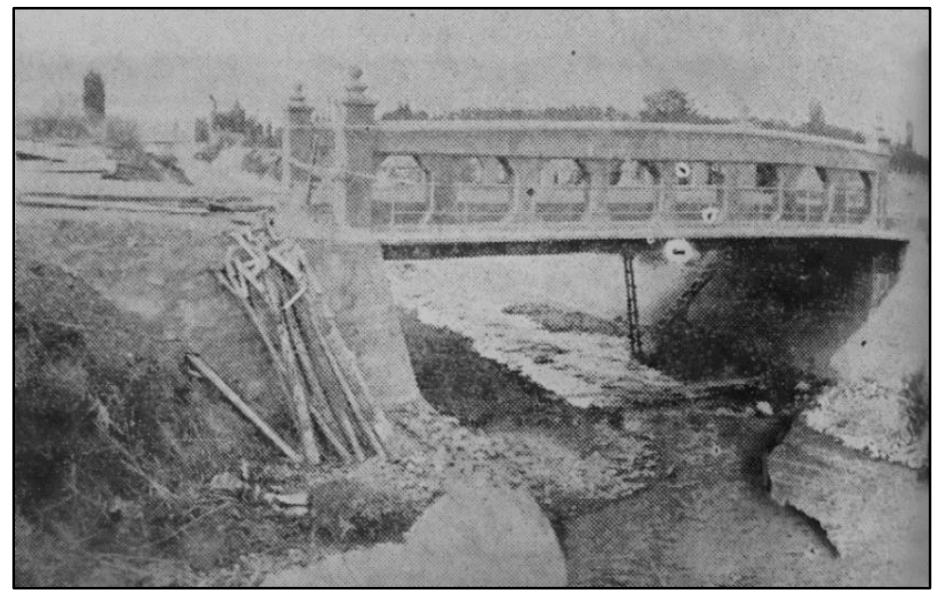

Fuente: Los Andes, 10/9/1928

Del mismo modo, como vía de unión entre Capital y Godoy Cruz, los vecinos de este último solicitaron que se levantara un puente. A fin de dar una respuesta, se propuso un

\footnotetext{
${ }^{81}$ Los Andes, 11/10/1928.
} 
proyecto de ley (aprobado en la Cámara de diputados el 31 de agosto de 1923) autorizando a construirlo en la intersección de la calle Paso de Los Andes y el Zanjón Frías. La obra se inauguró varios años después, el 28 de octubre de 1928. Así, mediante los ejemplos antedichos se puede observar el papel de los vecinos, que también cumplieron un papel importante a la hora de proponer y ejecutar obras viales.

Por otro lado, en los primeros años de la década de 1920 fue prioridad para los dirigentes el departamento de Lavalle, cuyos caminos estaban averiados debido al terremoto que había azotado al distrito en diciembre de 1920. Esto se veía agravado por las lluvias, que tornaban casi intransitable la vía hacia el departamento. La misma se reparó en 1923 (ver ilustración 7). Además, en diciembre del mismo año se inauguró un puente carretero sobre el arroyo Tulumaya (Lavalle).

Ilustración 8-Camino a Lavalle reconstruido en 1923

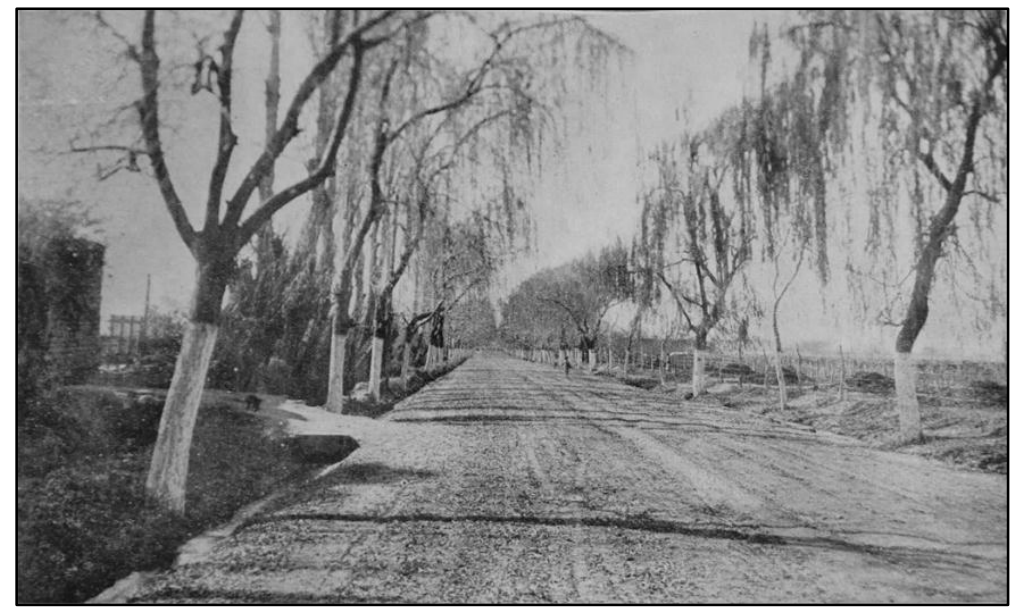

Fuente: Memoria del Ministerio de Obras Públicas. Ingeniero Leopoldo Suárez, 1922-1923

En Guaymallén fue inaugurada una obra el 11 de noviembre de 1923 en el paraje denominado "Paso de la Muerte". La misma constaba de un dique, un canal de desagüe y un sólido puente (ver Ilustración 8). En relación con la obra, Leopoldo Suárez (ministro de obras públicas) indicaba: "se ha llevado a cabo sujeta a un criterio científico, lo que le asegura magníficas condiciones de durabilidad y perfección, que evitarán los perjuicios que causaban las crecientes". ${ }^{82}$ De esa manera, el ingeniero indicaba cómo el conocimiento científico y los cálculos técnicos eran necesarios para garantizar la seguridad de la obra. En este sentido, puede observarse cómo los conocimientos de ingeniería eran claves para esa dependencia estatal.

\footnotetext{
${ }^{82}$ Leopoldo Suárez, Memoria presentada a la honorable legislatura por el ministro de industrias y obras públicas Ingeniero Leopoldo Suárez. Año 1922-1923, (Mendoza, 1924), pp.276.
} 
Ilustración 9- Puente sobre el "paso de la muerte" (Guaymallén). 1923

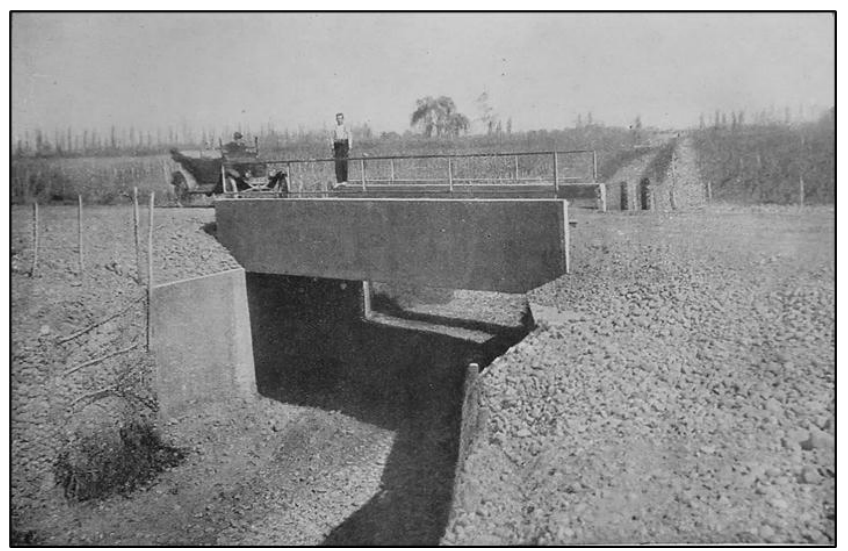

Fuente: Memoria del Ministerio de Obras Públicas. Ingeniero Leopoldo Suárez, 1922-1923

Además, en mayo de 1923 el gobierno ordenó sistematizar el camino comprendido entre la estación Negro Quemado, y el Puente sobre el río Atuel, "propendiendo a asegurar todo género de facilidades al desarrollo de las industrias". ${ }^{83}$ De la misma manera, se sistematizó el camino comprendido entre las estaciones de Agrelo y Ugarteche.

Por otro lado, los dirigentes sancionaron leyes y decretos relativos a la reconstrucción y reparación de otros tantos caminos. Un tramo del carril nacional en Rodeo de la Cruz, el carril a Luján, entre otros.

Ilustración 10- Ensanchamiento y nuevas vías de comunicación en capital

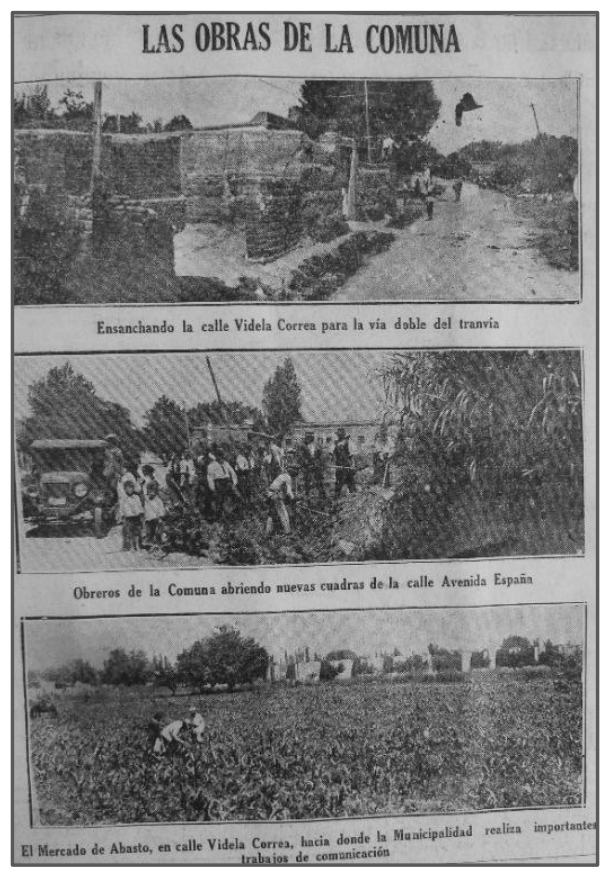

Fuente: La Palabra, 2 de enero de 1928

\footnotetext{
${ }^{83}$ Suárez, Memoria presentada..., p 284.
} 
Además, se hicieron obras de prolongación y ensanchamiento de calles (Montevideo, Rivadavia, Sarmiento, Videla Correa, entre otros caminos de la capital, y diversas calles pertenecientes a los departamentos) dando una respuesta a la nueva realidad urbana que, a partir del incremento poblacional y la expansión del transporte automotor principalmente, requería esas transformaciones (ilustración 10).

\section{Consideraciones finales}

En este estudio preliminar sobre la red vial en Mendoza en la década del '20 se pudo observar que la Dirección de Puentes y Caminos, dependencia estatal encargada de la vialidad provincial, se fue dotando de recursos financiaros y fue acudiendo más asiduamente a técnicos para los proyectos y ejecución de obras viales, los cuales fueron siendo paulatinamente incorporados a la burocracia estatal. De esa manera, la articulación entre técnica y política fue fortaleciendo la intervención estatal en materia vial, proceso que en la década de 1930 apareció más consolidado principalmente a partir de la creación de la Dirección Provincial de Vialidad en $19333^{84}$

Además, durante el periodo se implementaron nuevas técnicas y materiales que mejoraron la durabilidad y resistencia de las obras viales. De hecho, en los proyectos de ley sobre erección de puentes, se estipulaba que la construcción de estos se haría de manera sismorresistente.

Tal como se pudo observar, en la época de estudio la Dirección de Puentes y Caminos cumplió un papel fundamental a la hora de crear vías comunicacionales y adaptar las existentes para hacer frente a la nueva realidad de tránsito vehicular dada en la provincia (y en el país en general) debido a la expansión del parque automotor. Además, en las obras viales actuaron diferentes jurisdicciones, el Estado Nacional, el Estado Provincial y las municipalidades. Asimismo, en algunas ocasiones los vecinos cumplieron un papel significativo a la hora de solicitar y de financiar la construcción de vías de comunicación.

Durante el periodo, los dirigentes lencinistas propusieron y ejecutaron obras viales tendientes a mejorar la conexión interdepartamental a los fines de conectar la provincia y unir zonas alejadas. La argumentación a favor de las obras giró en torno al progreso y las facilidades comerciales que estas traerían aparejadas, particularmente como medio para facilitar el traslado durante la época de cosecha. Asimismo, los dirigentes propusieron mejorar

\footnotetext{
${ }^{84}$ Para ampliar información consultar: Raffa y Luis, Caminos para el turismo...
} 
los caminos para fomentar el desarrollo turístico de la provincia, sobre todo del área montañosa.

Por otro lado, durante el lapso temporal abordado aparecieron y se extendieron nuevos medios de transporte que modificaron la realidad urbana de Mendoza y requirieron de mayor intervención estatal a los fines de regular el tránsito y las condiciones que debían cumplir los vehículos y conductores. Asimismo, facilitaron el arribo de los sectores de clase media y popular a lugares antes inaccesibles. ${ }^{85}$

Resta avanzar en investigaciones posteriores en el estudio de trayectorias individuales relevantes durante el periodo que fueron fundamentales a la hora de proyectar y ejecutar obras viales y pasaron a formar parte de la burocracia técnica provincial.

\footnotetext{
${ }^{85}$ Cfr: Luis, automovilismo y transformación urbana...
} 